\title{
Research Article \\ The Study of the Chaotic Behavior in Retailer's Demand Model
}

\author{
Junhai Ma and Yun Feng \\ School of Management, Tianjin University, Tianjin 300072, China \\ Correspondence should be addressed to Junhai Ma, mjhtju@yahoo.com.cn
}

Received 26 February 2008; Accepted 14 September 2008

Recommended by Weihong Huang

Based on the work of domestic and foreign scholars and the application of chaotic systems theory, this paper presents an investigation simulation of retailer's demand and stock. In simulation of the interaction, the behavior of the system exhibits deterministic chaos with consideration of system constraints. By the method of space's reconstruction, the maximal Lyapunov exponent of retailer's demand model was calculated. The result shows the model is chaotic. By the results of bifurcation diagram of model parameters $k, r$ and changing initial condition, the system can be led to chaos.

Copyright (c) 2008 J. Ma and Y. Feng. This is an open access article distributed under the Creative Commons Attribution License, which permits unrestricted use, distribution, and reproduction in any medium, provided the original work is properly cited.

\section{Introduction}

A supply chain is a complex system which involves multiple entities encompassing activities from the raw material to the final delivery stage. A simple supply chain system includes not only supplier, manufacturers, retailers, and customers, but also all the flow of information and funds. So there exist various types of uncertainties along the chain, for example, demand uncertainty, production uncertainty, and lead time uncertainty. As a result, the supply chains are much more dynamic. Such a dynamic and complex environment presents a big challenge for researcher to handle uncertainty in an efficient and effective way. One type of uncertain behaviors is bullwhip effect [1], which increases the variability of demand in the supply chain. Lee et al. [2] identified four uncertain causes of the bullwhip effect: demand forecast, reorder batching, pricing fluctuation, and shortage gaming. Later, Chen et al. [3] quantify the effect in a simple two-stage supply system with statistics method and AR(1) demand process. However, statistic method only deals with continuous time and linear problems, the uncertain activities generate interactions among customers, and suppliers usually lead to discrete time and nonlinear dynamic behaviors or even chaos. Many researches focus on the uncertainty field with chaotic theory.

Chaos is disorderly looking for long-term evolution occurring in a deterministic nonlinear system. Chaos theory is concerned with chaos behavior in nonlinear dynamical systems from a number of aspects. The origin of chaos theory dates back to Lorenz's [4] study 
in weather forecasting system. Subsequently, many researchers have contributed to greater interest in studying and applying chaos theory to inventory, management, and supply chain studies. It was reported that a chaotic demand for liquid detergent exists at the interface of a manufacturer and its distributors [5]. Kumara et al. [6] demonstrated that in a queuing model of supply chain logistics systems, the behaviors of the system exhibit chaos. Wang et al. [7] explore an efficient approach for identifying chaotic phenomena in demands and develops a production lot-sizing method for chaotic demands. Makui and Madadi [8] use the maximal Lyapunov exponent, a chaos parameter, to measure the bullwhip effect and get some useful results on the behavior of the bullwhip effect. Wu and Zhang [9] present an investigation of interaction between customers and suppliers in a three-tier supply chain system, and found that the attractors of the model move with the environment and the initial states. Hwarng and Xie [10] investigate how this class of variability, chaos, may occur in a multilevel supply chain and offer insights into how to manage relevant supply chain factors to eliminate or reduce system chaos.

A system of chaos is often characterized by a number of distinct features, for example, nonrandomness and nonlinearity, apparent disorder: the motion of the variables looks disorganized and irregular; strange attractor: pattern can be found in phase space; and sensitivity to initial conditions: a small change in initial conditions can have a large effect on the evolution of the system.

Chaotic behaviors can be either qualitatively identified by figure patterns methods which identify chaos or show whether a system is stable, periodic, quasiperiodic, or chaotic, such as Poincare map, phase plots, and power spectrum. Graphs and plots are visually efficient in showing trends and patterns. Another more accurate alternative is to calculate some quantifies, such as capacity dimension, correlative dimension, Kolmogorov entropy, and the maximal Lyapunov exponent. For example, provided the maximal Lyapunov exponent is a positive number, the investigated dynamic model is likely to be chaotic.

This paper is concerned with the model of retailer's demand and calculates the system chaotic parameter which decides the chaotic system. By plotting the bifurcation diagram, the parameter of model can lead the system to the chaotic behavior.

\section{Demand model}

There were various supply chain models studied previously. Most of them were based on a simple beer distribution model [11] which includes three levels: brewery or factory, wholesaler, and retailer. In this system, orders propagate from customers to factory. In order to distinguish the traditional beer model, this paper focuses on the model of retailer's demand decision and study the complex behavior between retailer and the whole supply chain. The model is constructed on a customer-supplier system, including customers, a retailer, and a manufacturer. The customers buy products from the retailer. The demand of the customers depends on the price offered by the retailer. The retailer forecasts the demand based on the previous sales and makes the new order to the manufacturer accordingly. The manufacturer makes only what the retailer is ordered. However, the manufacturer does not accept the order with quantity in excess of its capacity. The retailer determines the sales price for the next period according to the current stock. If the stock level is high, the retailer offers a discount to encourage the customer to buy more. The process is deterministic on the basis of settled rules or formulae and repeats automatically in the next period.

It is assumed that the amount of the stock increase is the difference of the amount received, which is ordered in the previous period, and the amount of sales to customers as 
given by Steman [12]. That is,

$$
S_{t+1}=q_{t+1}+S_{t}-D_{t}
$$

where $D_{t}$ is the actual demand at $t$ period. $q_{t+1}$ is the order from retailer to manufacture and is equal to $P_{t+1}$ which is the output of manufacturer at $t$ period.

It is assumed [12] that the retailer sends a request for quotation of the product in a quantity as forecasted. On receiving the request, the manufacture plans the production and accepts the order with limited production capacity in the period. If the production capacity is infinite, the $P_{t+1}$ should be equal to the expected demand $\widehat{D}_{t+1}$ which is the expected demand of customer in next period. Then,

$$
S_{t+1}=\widehat{D}_{t+1}+S_{t}-D_{t}
$$

The retailer forecasts the demand by simple exponential smoothing by Towill [13], the expected demand at time $t$ can be defined as follows:

$$
\widehat{D}_{t}=\omega D_{t-1}+(1-\omega) \widehat{D}_{t-1}, \quad 0<\omega<1,
$$

where $D_{t}$ is the actual demand of customer. $\omega$ is an exponential constant which determines how fast expectations are updated; when $\omega=1$, the expected demand $\widehat{D}_{t}$ at $t$ period is equal to the actual demand $D_{t-1}$ at $t-1$ period. At the $\omega=0, \widehat{D}_{t}=\widehat{D}_{t-1}$, the actual demand can be ignored.

If the new stock level $S_{t+1}$ excesses a threshold, $T$, the retailer regards the level as overstock and prepares to make a discount offer to reduce the stock. An overstock rate $v_{t+1}$ is calculated as

$$
v_{t+1}=\frac{S_{t+1}-T}{T} .
$$

The parameter $T$ is a policy parameter for the retail to evaluate the stock level and determine whether to offer a price discount or not. Price discount is an effect behavior to alleviate the inventory's pressure and magnify the customer's demand at short time. But the discount offer is only valid for one period. The discount for the next period will be re-evaluated by the overstock rate at the end of the period. The relationship between overstock rate and discount rate is hence calculated by

$$
r_{t+1}=\frac{v_{t+1}}{q},
$$

where $q$ is a constant. When the $r_{t+1}$ increases, the price will be decreased. As a result, the customer's demand will increase. The trend of demand's increase is the result of the retailers' active behaviors and avoids the venture which changes from the uncertain demand to actual inventory.

Because the customers are myopic, the customer's demand $R_{t+1}$ is deterministic by the price. If there is no discount, the customer' demand is equal to the last period actual demand 
$D_{t}$, referred to as basic demand. When a discount rate is offered, the demand increases based on the basic demand [9]:

$$
R_{t+1}=\frac{D_{t}}{\left(1-r_{t+1} / a\right)^{k}}
$$

where $a$ is the upper limit of the discount, $k$ is a parameter which determines the demand under discount, $0<k<1$. If not considering the price fluctuation, the customer's demand $R_{t+1}$ is equal to the retailer's actual demand $D_{t+1}$ among the linear models of demand. But the linear relationship cannot describe the dynamic demand model more realistic, many researchers prefer the nonlinear function for the demand model. According to the model of Qi et al. [14], the market's actual demand is a downside nonlinear function of retail price:

$$
D_{t+1}=D p^{-2 r},
$$

where $r$ is a coefficient of price sensitivity, $D$ is the maximum market scale and presents the customer's forecast demand in market. So the maximum scale $D$ is equal to the customer's demand $R_{t+1}$.

From (2.6) and (2.7), the actual demand and price can be expressed as

$$
D_{t+1}=\frac{D_{t} p^{-2 r}}{\left(1-r_{t+1} / a\right)^{k}} .
$$

Form (2.4), (2.5), and (2.8),

$$
D_{t+1}=\frac{(q a T)^{k} p^{-2 r}}{\left[(q a+1) T-S_{t+1}\right]^{k}} D_{t} .
$$

The model above can be expressed as a high-demand piecewise map in the following mathematical form:

$$
\begin{aligned}
& x_{t+1}=\frac{(q a T)^{k} p^{-2 r} x_{t}}{\left[(q a+1) T-y_{t+1}\right]^{k}}, \\
& y_{t+1}=y_{t}+z_{t+1}-x_{t}, \\
& z_{t+1}=\omega x_{t}+(1-\omega) z_{t} .
\end{aligned}
$$

\section{Chaos's identifying method}

A simple chaotic system has two characters: sensitivity to initial conditions and the strange attractor with fractal structure. For the model (2.10), this paper uses the time series of retailer's actual demand to reflect the system behavior. The chaotic character can be identified through calculating the embedding dimension of a scalar time series. If we only consider one variable and make it invariable based on Cao [15] theorem, an equal reconstructed space will be identified from the new point which is taken as the new dimension on the time delay point. 
When embedding dimension accumulates to a new level, the reconstructed space has all the same dynamic characters as the actual system and also does not rely on the reconstructed detail of process.

The method presented in this paper supposes a time series. The time-delay vectors can be reconstructed as follows:

$$
X_{n}=\{x(n), x(n+\tau), \ldots, x(n+(m-1) \tau)\}, \quad n=1,2, \ldots, N-(m-1) \tau,
$$

where $m$ is the embedding dimension and $\tau$ is the time delay. Note that $X_{n}$ means the $i$ th reconstructed vector with embedding dimension $m$. Similar to the idea of the false neighbor method, it is defined as follows:

$$
a(i, m)=\frac{\left\|X_{N N+1}-X_{n+1}\right\|^{m+1}}{\left\|X_{N N}-X_{n}\right\|^{m}}, \quad i=1,2, \ldots, N-m \tau,
$$

where $\|\cdot\|$ is some measurement of Euclidian distance and is given in this paper by the maximum or the mean value of all $a(i, m)$ 's:

$$
E(m)=\frac{1}{N-m \tau} \sum_{i=1}^{N-m \tau} a(i, m)
$$

where $E(m)$ is dependent only on the dimension $m$ and the lag $\tau$. To investigate its variation from $m$ to $m+1$, let $E 1(m)=E(m+1) / E(m)$. However, $E 1(m)$ stops changing when $m$ is greater than some value $m_{0}$; if the time series comes from an attractor, then $m_{0}+1$ is the minimum embedding dimension.

Before numerical tests, it is necessary to define another quantity which is useful to distinguish deterministic signals from stochastic signals. Let

$$
E^{*}(m)=\frac{1}{N-m \tau} \sum_{i=1}^{N-m \tau}|x(i+m \tau)-x(N N+m \tau)|
$$

where the meaning of $(N N+m \tau)$ is the same as above, that is, it is the integer such that $a(i, m)$ is the nearest neighbor of $a(i, m)$. Let

$$
E 2(m)=\frac{E^{*}(m+1)}{E^{*}(m)}
$$

For time series data from a random set of numbers, $E 1(m)$, in principle, will never attain a saturation value as $m$ increases. But in practical computations, it is difficult to resolve whether the $E 1(m)$ is slowly increasing or has stopped changing if $m$ is sufficiently large. In fact, since available observed data samples are limited, it may happen that the $E 1(m)$ stops changing at some $m$ although the time series is random. It is recommended calculating both $E 1(m)$ and $E 2(m)$ for determining the minimum embedding dimension of a scalar time series, and distinguishing deterministic data from random data. 


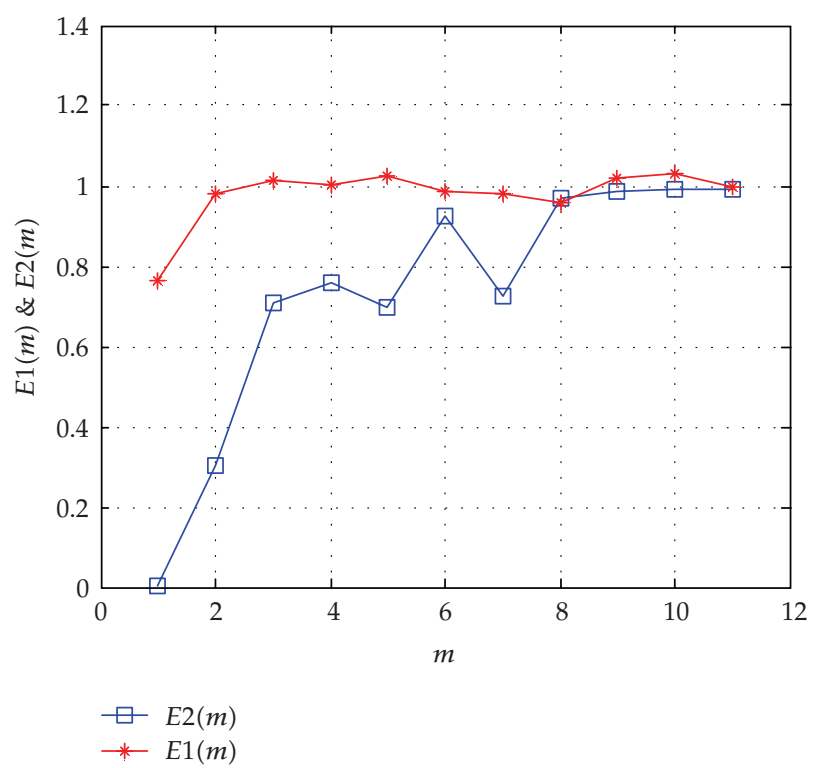

Figure 1: The values $E 1(m)$ and $E 2(m)$ for the data from the retailer's model.

Above on the describe, we numerate the model (2.10) with step 0.01, and record the time series data from the numerical solution with sampling time 6 after all transients have been diminished. The results for this time series are shown in Figure 1, where $\tau=1$, sampling time. One can see that the $E 1(m)$ attains its saturation value at $m=2$ and the $E 2(m)=1$ at $m=8$, it shows that the time series is chaotic series. Therefore, $m=2$ should be the minimum embedding dimension for the time series in our model. Obviously, the result does not strongly depend on how many data points are used. Because the $E 1(m)$ attains its saturation at $m=2$, the models of retail's demand have clearly chaotic behaviors.

After getting the embedding dimension of time series, it is necessary to calculate the maximal Lyapunov exponent for the chaotic model. It is assumed that the retailer's demand, which is the origin of (2.10), is located in a reconstructed space like (3.1). The analysis presented here first identifies the starting point $x_{j}$ (in the space) which is closest to the initial reference point $x_{i}$. The distance between the two points is defined as

$$
d_{j}\left(x_{i}, x_{j}, t\right)=\left|x_{j+m-1+t}-x_{i+m-1+t}\right| .
$$

Let

$$
S(t)=\frac{1}{N} \sum_{j=1}^{N} \operatorname{In}\left(\frac{1}{\left|U_{j}\right|} \sum_{i \in U_{j}} d_{j}\left(x_{j}, x_{i}, t\right)\right),
$$

where $U_{j}$ is the E-neighborhood included $x_{i}, x_{j}$. When $S(t)$ increase as the $t$, the regression curve will be plotted by the method of least squares. Calculating the slope of regression curve in the $S(t)-t$ plot and the value of slope is the maximal Lyapunov exponent.

Figure 2 shows the maximal Lyapunov exponent of retailer's demand. In the $S(t)-t$ plot, the slope of $S(t)$ 's curve equals 0.05297 . It clearly confirmed that the dynamic system 


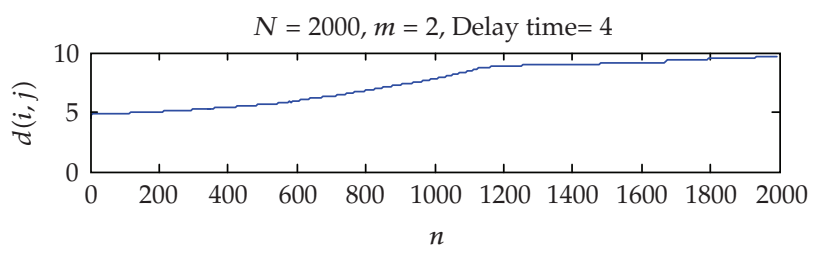

(a)

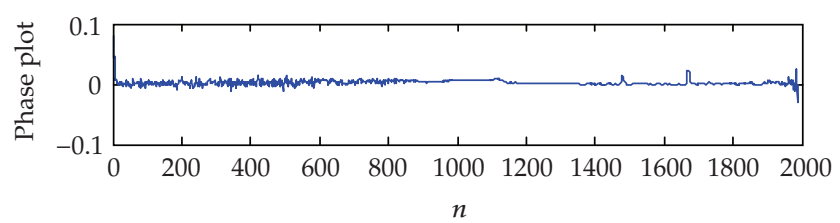

(b)

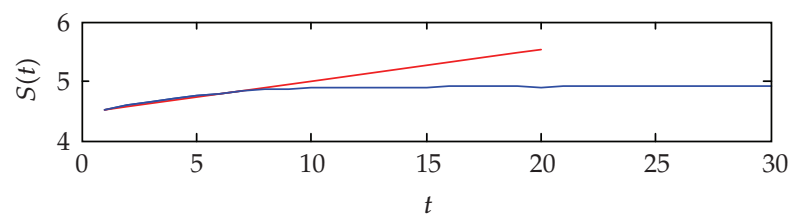

(c)

Figure 2: The maximal Lyapunov exponent of retailer's demand.

Table 1: The parameters and initial data.

\begin{tabular}{lc}
\hline Item & Value \\
\hline Initial retailer's demand, $D_{0}$ & 500 \\
Initial stock, $S_{0}$ & 1000 \\
Initial expected demand $\widehat{D}_{0}$ & 0 \\
Discount threshold, $T$ & 600 \\
Ratio of overstock, $q$ & 1.2 \\
Discount, $a$ & $70 \%$ \\
Exponential constant, $\omega$ & 0.82 \\
$p^{-2 r}$ & 1 \\
\hline
\end{tabular}

based on (2.10) has chaotic behavior. In the next step, simulation of the model is necessary to prove that the chaotic behavior is sensitive to initial conditions.

\section{Simulation results and discussion}

The initial data, as show in Table 1, for running the model described above were chosen arbitrarily. But the value of price must be equal to 1, it easy to find the relationship between the actual stock and expected demand.

Because the flow of demand or stock cam reflects the operational complexity of system, the investigation of the system behavior in the paper will concentrate on the stock and demand held by retailer. All the bifurcation diagrams in the following, wherever it is not 


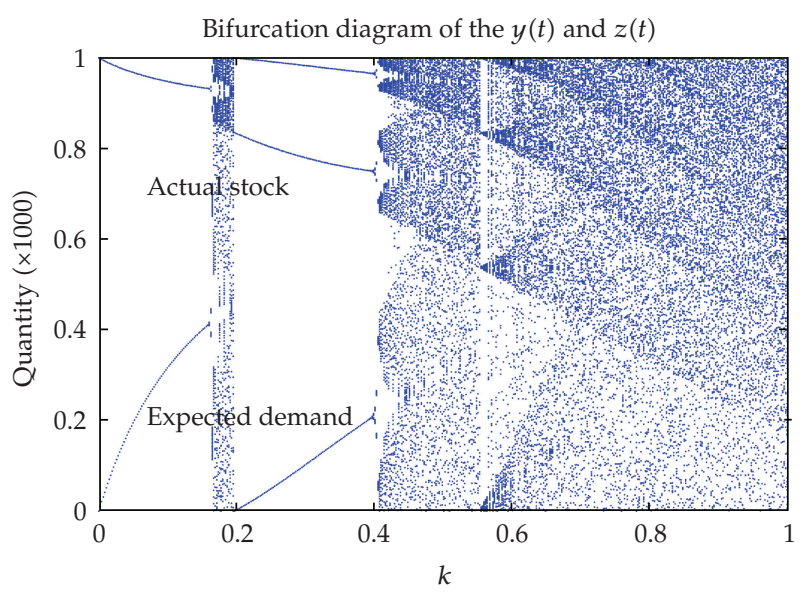

Figure 3: The bifurcation diagram of actual stock and expected demand.

specified, were generated with a series time of 10000 iterations. Figure 3 shows the chaotic behavior of retail's actual and expected demand, which is based on (2.10). It is clear in the bifurcation diagram that the dynamic system does not attain an equilibrium state in the simulated interval between 0.1 and 1 of parameter $k$. Aperiodic behavior happens in two intervals $(0.16,0.19)$ and $(0.404,1)$ and the stock and demand appear to be periodically oscillatory in the intervals from 0.1 to 1.0 .

\subsection{Impact of the price fluctuation}

The above results show that the retailer's demand model based on (2.10) under the given conditions, including initial states as described previously, will go into chaos in certain ranges of $k$. So it is necessary to investigate the impact of the price fluctuation on the system. Based on formula (2.7), $D_{t+1}=D p^{-2 r}$, the retailer's price is decided by the parameter $r$ which is the exponential forms. The results of simulation of exponential parameter $r$ and retailer's demand are shown in Figure 4. It is clearly chaotic behavior in retailer's demand as the $r$ increases. When $r$ varies from 0.48 to 1.2 , the retailer's demand shows the chaotic behavior. At $r=0.48$, the period doubling bifurcation which appeared and decreased the initial $r$ to 0.14 will bring the system shifts from oscillation to equilibrium.

When price decreases, it is normal that the customer's demand will increase. If the amount of stock is enough to satisfy the demand, which is called no stockout, the demand is positive like the diagram at $r \in(0,0.48)$ in Figure 4 . When $r>0.48$, the demand is negative, it obviously means that the stockout happened. As a result, the system of (2.10) shows chaotic behavior. So the stockout is the uncertain behavior and can lead the system to the chaos. Please refer to the Xu et al.'s article [16] for the further discussion about the coefficient of the price sensitivity $r$.

The above results show that $r>0.48$ would lead the system into chaos and stockout. It is necessary to discuss the equilibrium point when the stockout does not happen. Figure 5 shows the retail's stock bifurcation as the $r$ changes and the system of (2.10) shows chaotic behavior. When the coefficient $0.41>r>0.48$, the retail's stock into oscillation and $r>0.48$, the system from oscillation to chaos. 


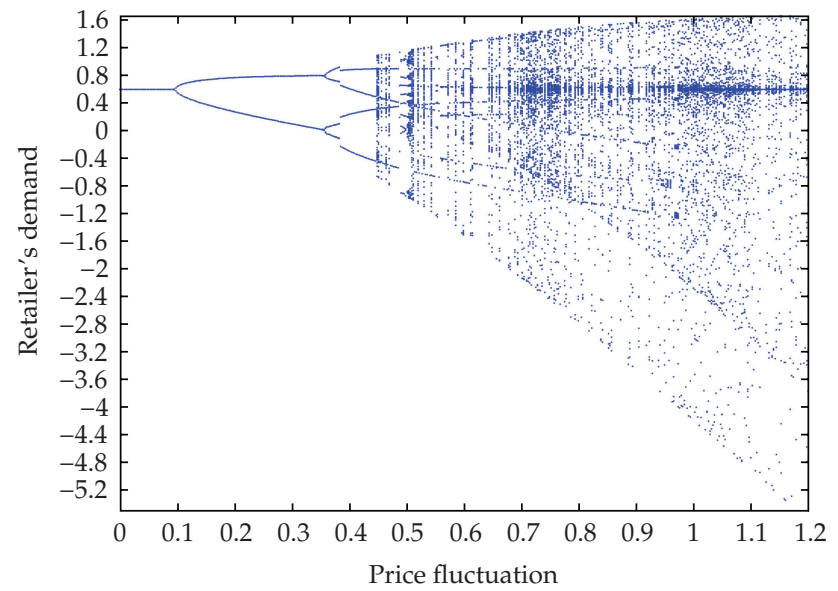

Figure 4: The bifurcation diagram of retail's demand $(k=0.6, p=10)$.

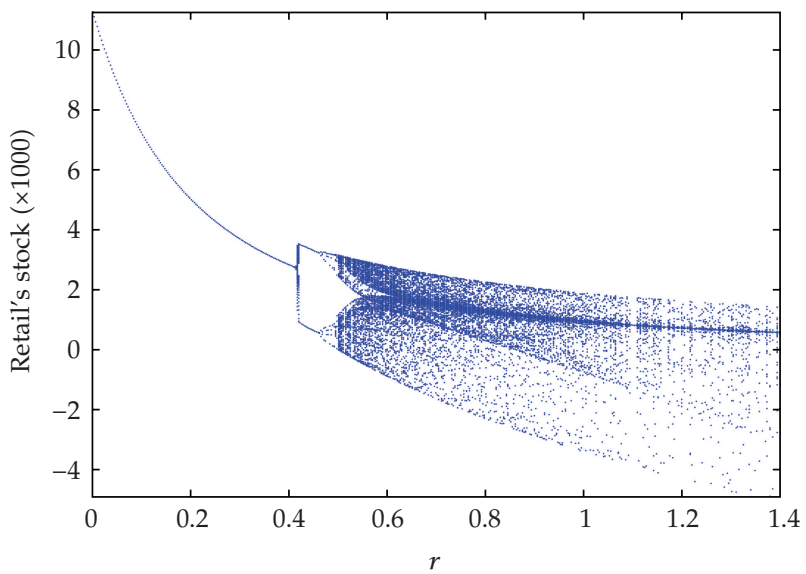

Figure 5: The bifurcation diagram of retail's stock $(k=0.6, p=10)$.

Whether the stockout happened or not, according to Figures 4 and 5, $r>0.48$ can lead the system of (2.10) to chaos. Because coefficient of price sensitivity $r$ is decided by

$$
D_{t+1}=D p^{-2 r},
$$

$r>0.48,2 r>1, p^{2 r} \geq p^{1}$, the price is not a fluctuation at $2 r=1$. As an increase of $r$ from 0.48 , the price fluctuation gets worsened, the stockout and chaos happened. The system of (2.10) shows the chaotic behavior.

\subsection{Impact of the initial condition}

It is well known [17] that attractors of a high-order map can be sensitive to initial conditions. Any change of initial stock or demand can be regarded as a disturbance of system and can lead to chaos. Figure 6 shows the bifurcation diagrams when the initial stock increases by $5 \%$ 


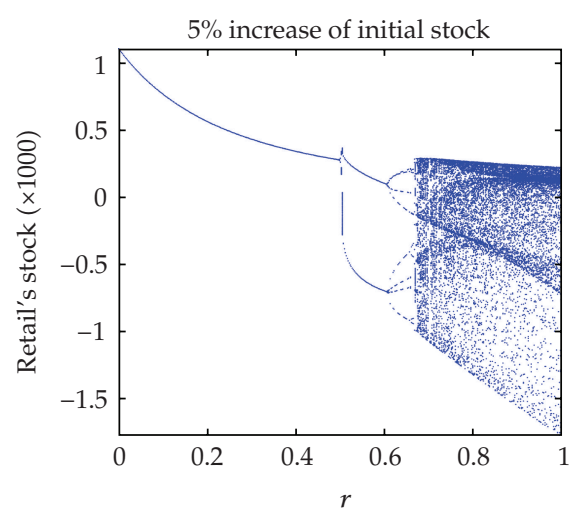

(a)

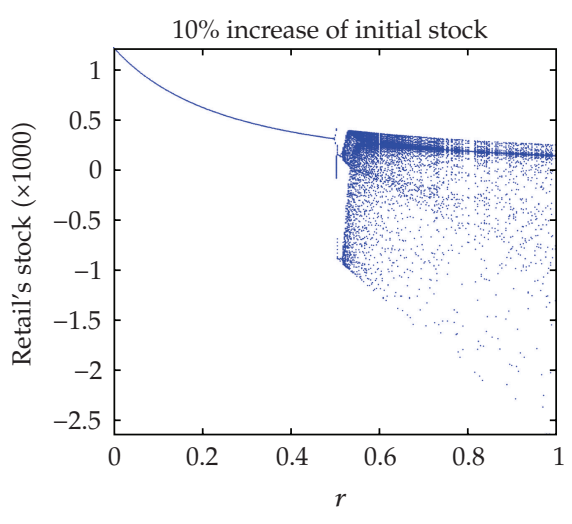

(b)

Figure 6: Bifurcation diagram of 5\% and $10 \%$ increase of initial stock.

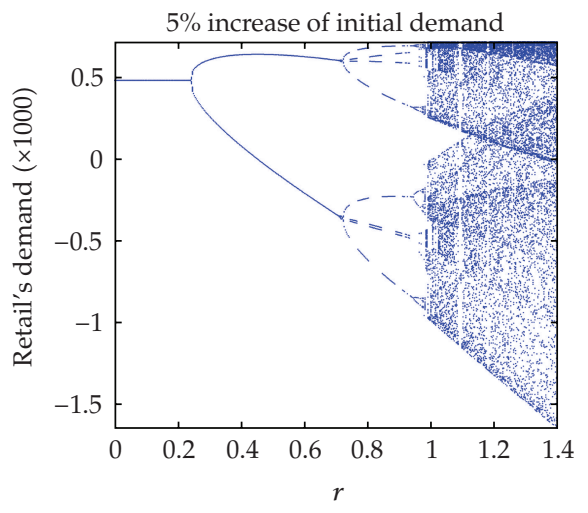

(a)

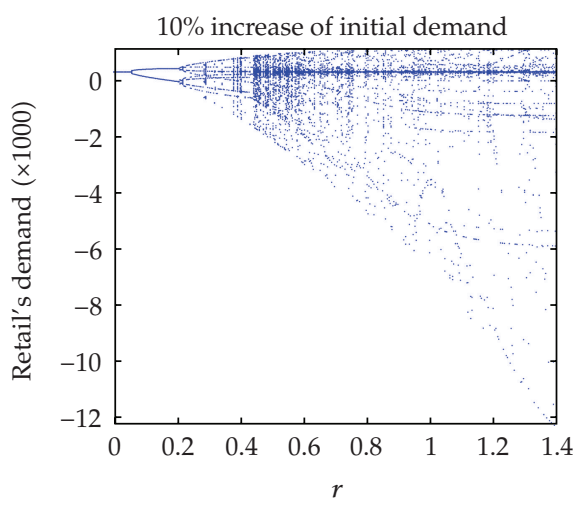

(b)

Figure 7: Bifurcation diagram of 5\% and 10\% increase of initial demand.

and $10 \%$, respectively. In the case of initial stock increase by $5 \%$, the major aperiodic interval moves to 0.68 . In the case of initial stock increase by $10 \%$, the bifurcation diagram shows much less chaotic behavior. It should be noted that the change of initial stock also caused variance of the bifurcation diagram sharps.

The impact of other initial data was also investigated. Figure 7 shows the bifurcation diagrams when the initial demand increases by $5 \%$ and $10 \%$, respectively. It is clear that the change of initial demand also leads the system to chaos.

The above results show that the system state and the behavior depend not only on the model's parameters, but also on the initial state. As a state-at any time-can be an initial state for future evolution, the behavior of such a system is thus sensitive to disturbances. A disturbance to the system states could eventually lead the system into equilibrium or chaos.

\subsection{Adaptive adjustment}

Despite the model of retailer's demand shows the chaotic behavior, the mechanism of stabilizing the chaotic process in the actual supply chain is still under discussion. In the 
dynamic system, Huang and Zhang [18] advanced the theory of the adaptive adjustment which is the effective method to smooth the chaotic behavior. In Huang's discussion [19], conventional adaptive expectations as a mechanism of stabilizing an unstable economic process are reexamined through a generalization to an adaptive adjustment framework. The generic structures of equilibrium that can be stabilized through adaptive adjustment mechanisms are identified theoretically and numerically. The adaptive adjustment schemes can be applied to a broader class of discrete economic processes, such as in cobweb dynamics, price adjustment, tariff game, population control model.

\section{Conclusion}

This paper has presented a model of retailer's demand. In simulation of the interaction, the behavior of the system exhibits deterministic chaos with consideration to system constraints. By the method of space-reconstructed, this paper calculates the minimum embedding dimension and the maximal Lyapunov exponent of retailer's demand model. The result shows that the model is chaotic. In the last, simulations have demonstrated that the parameters $k$ and $r$ can lead the system to the chaotic behavior.

As a result of simulation, the model exhibits different behaviors as the initial condition varies. The sensitivity of the chaotic behavior to initial conditions makes the system easy to be disturbed in a changing environment. In reality, the system keeps disturbed all the time by various factors and the disturbance could be amplified in this way. Thus, the later state of the system remains unpredictable.

The simulation model discussed in this paper can inflect that the real supply chain is much more complex and its behavior is hence much more complicated. The managers should smoothen the uncertain behavior in supply chain. As a result, the behavior of the system can be much less chaotic than simulated.

\section{References}

[1] J. W. Forrester, Industrial Dynamics, The MIT Press, Cambridge, Mass, USA; John Wiley \& Sons, New York, NY, USA, 1961.

[2] H. L. Lee, V. Padmanabhan, and S. Whang, "Information distortion in a supply chain: the bullwhip effect," Management Science, vol. 43, no. 4, pp. 546-558, 1997.

[3] F. Chen, Z. Drezner, J. K. Ryan, and D. Simchi-Levi, "Quantifying the bullwhip effect in a simple supply chain: the impact of forecasting, lead times, and information," Management Science, vol. 46, no. 3, pp. 436-443, 2000.

[4] E. N. Lorenz, "Deterministic nonperiodic flow," Journal of the Atmospheric Sciences, vol. 20, no. 2, pp. 130-141, 1963.

[5] Y. Wu, G. Frizelle, L. Ayral, J. Marsein, E. Van de Merwe, and D. Zhou, “A simulation study on supply chain complexity in manufacturing industry," in Proceedings of the Conference of the Manufacturing Complexity Network, University of Cambridge, Cambridge, UK, April 2002.

[6] S. R. T. Kumara, P. Ranjan, A. Surana, and V. Narayanan, "Decision making in logistics: a chaos theory based analysis," CIRP Annals: Manufacturing Technology, vol. 52, no. 1, pp. 381-384, 2003.

[7] K.-J. Wang, H.-M. Wee, S.-F. Gao, and S.-L. Chung, "Production and inventory control with chaotic demands," Omega, vol. 33, no. 2, pp. 97-106, 2005.

[8] A. Makui and A. Madadi, "The bullwhip effect and Lyapunov exponent," Applied Mathematics and Computation, vol. 189, no. 1, pp. 35-40, 2007.

[9] Y. Wu and D. Z. Zhang, "Demand fluctuation and chaotic behaviour by interaction between customers and suppliers," International Journal of Production Economics, vol. 107, no. 1, pp. 250-259, 2007.

[10] H. B. Hwarng and N. Xie, "Understanding supply chain dynamics: a chaos perspective," European Journal of Operational Research, vol. 184, no. 3, pp. 1163-1178, 2008. 
[11] W. E. Jarmain, Problem in Industrial Dynamics, The MIT Press, Cambridge, Mass, USA, 1963.

[12] J. D. Steman, "Modeling management behavior: misperceptions of feed back in a dynamic decision making experiment," Management Science, vol. 35, pp. 321-339, 1989.

[13] D. R. Towill, "Fundamental theory of bullwhip induced by exponential smoothing algorithm," MASTS Occasional Paper. Cardiff University, 1999.

[14] X. Qi, J. F. Bard, and G. Yu, "Supply chain coordination with demand disruptions," Omega, vol. 32, no. 4, pp. 301-312, 2004.

[15] L. Cao, "Practical method for determining the minimum embedding dimension of a scalar time series," Physica D, vol. 110, no. 1-2, pp. 43-50, 1997.

[16] M. Xu, X. Qi, G. Yu, H. Zhang, and C. Gao, "The demand disruption management problem for a supply chain system with nonlinear demand functions," Journal of Systems Science and Systems Engineering, vol. 12, no. 1, pp. 82-97, 2003.

[17] J. T. Sandefur, Discrete Dynamical Systems: Theory and Applications, The Clarendon Press, Oxford University Press, New York, NY, USA, 1990.

[18] W. Huang and Y. Zhang, "Distributional dynamics of cautious economic adjustment processes," Journal of Economic Behavior E Organization, vol. 62, no. 3, pp. 389-407, 2007.

[19] W. Huang, "Theory of adaptive adjustment," Discrete Dynamics in Nature and Society, vol. 5, no. 4, pp. 247-263, 2001. 


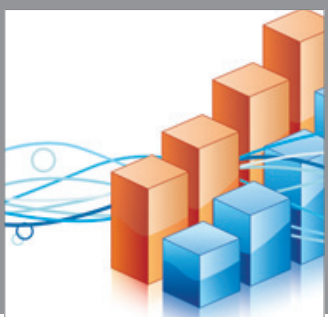

Advances in

Operations Research

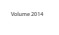

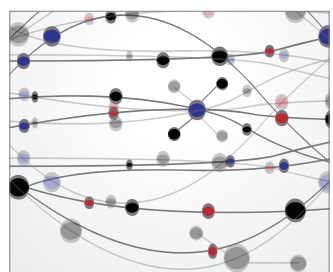

\section{The Scientific} World Journal
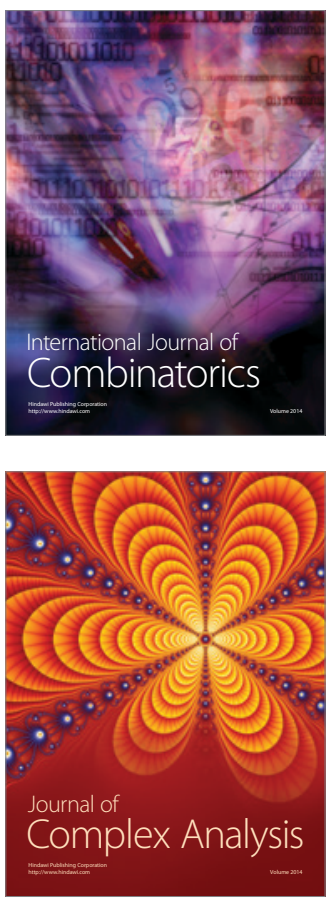

International Journal of

Mathematics and

Mathematical

Sciences
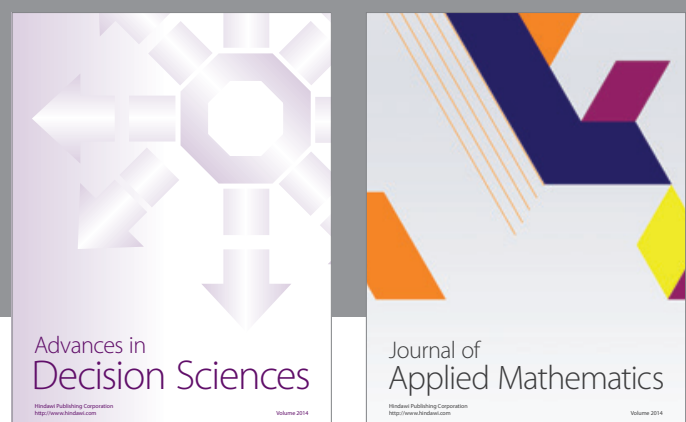

Journal of

Applied Mathematics
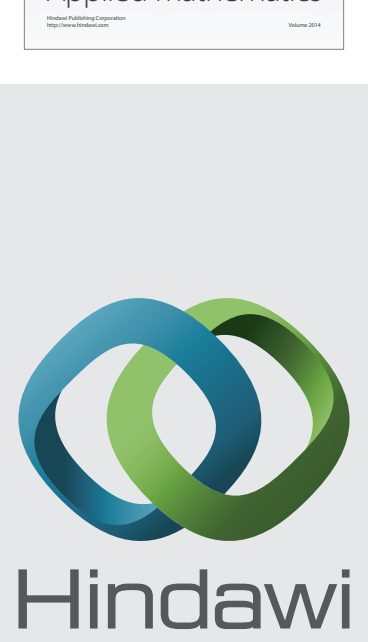

Submit your manuscripts at http://www.hindawi.com
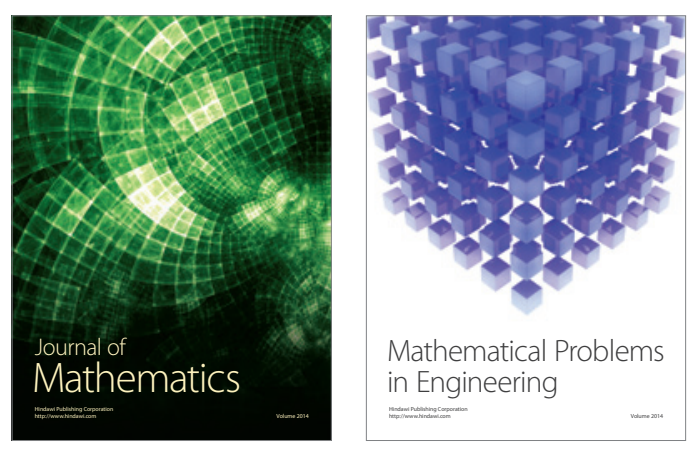

Mathematical Problems in Engineering
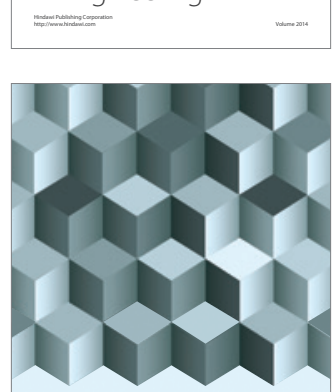

Journal of

Function Spaces
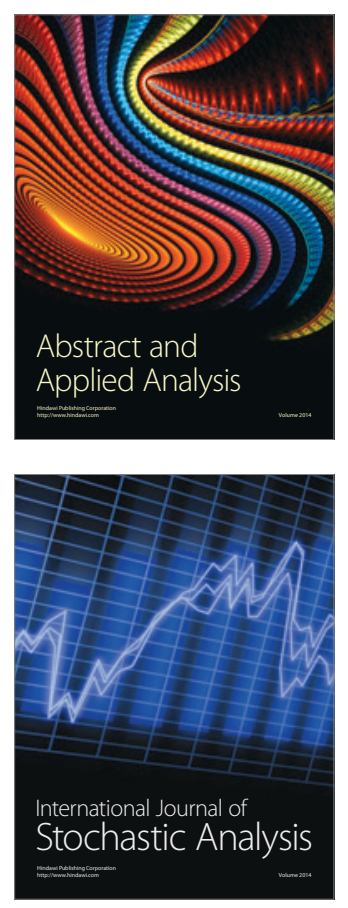

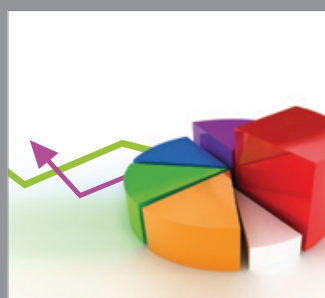

ournal of

Probability and Statistics

Promensencen
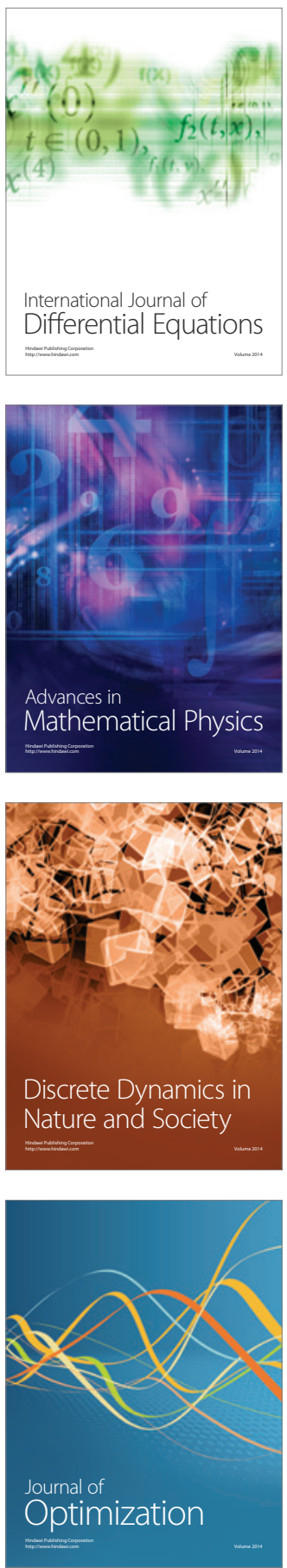